\title{
Pour une étude génétique du Livre des tables : les révélations du manuscrit Durrieu
}

Jean-Claude Fizaine

\section{(2) OpenEdition}

1 Journals

Édition électronique

URL : http://journals.openedition.org/genesis/2936

DOI : 10.4000/genesis.2936

ISSN : 2268-1590

Éditeur :

Presses universitaires de Paris Sorbonne (PUPS), Société internationale de génétique artistique littéraire et scientifique (SIGALES)

Édition imprimée

Date de publication : 5 décembre 2017

Pagination : 99-111

ISBN : 979-10-231-0580-3

ISSN : 1167-5101

\section{Référence électronique}

Jean-Claude Fizaine, "Pour une étude génétique du Livre des tables : les révélations du manuscrit

Durrieu », Genesis [En ligne], 45 | 2017, mis en ligne le 15 décembre 2018, consulté le 11 janvier 2021. URL : http://journals.openedition.org/genesis/2936 ; DOI : https://doi.org/10.4000/genesis.2936 


\title{
Pour une étude génétique du Livre des tables : les révélations du manuscrit Durrieu
}

\author{
Jean-Claude Fizaine
}

$\mathrm{L}$ ors de son séjour à Jersey, île de l'archipel francoanglais où il s'était réfugié en juillet 1852 avec sa famille après avoir été expulsé de France pour avoir participé à la résistance armée contre le coup d'État, Victor Hugo assista et participa à des séances dites «spirites », organisées collectivement chez lui entre le 11 septembre 1853 et le 8 octobre 1855. Cette pratique, importée d'Amérique puis introduite à Jersey par une visite de Mme de Girardin, consistait à évoquer les esprits de défunts proches ou illustres, avec qui on communiquait par des frappements produits par le trépied d'un guéridon. Ce qui était une curiosité ou un jeu mondain à Paris devint pour les exilés de Jersey réunis autour de Victor Hugo un puissant stimulant à la créativité collective. Les textes produits sous la direction de Charles, fils de Victor Hugo qui s'imposa très tôt comme le meilleur, voire le seul «médium», textes d'une qualité et d'une abondance remarquables, étaient soigneusement enregistrés dans des «procès-verbaux» qui finirent par constituer quatre cahiers, que le poète conserva par-devers lui avec le même soin qu'il apportait à ses propres manuscrits. Toutefois, ils furent soustraits à sa mort à l'inventaire général de ses manuscrits. Les textes publiés pour la première fois en 1923 sous le titre Les Tables tournantes de Jersey ${ }^{1}$ ont donc été édités à partir de copies faites par diverses personnes à diverses dates. Après cette publication partielle, deux éditions se sont succédé à mesure que certains cahiers refaisaient surface, l'une en 19682, l'autre en 20143, chaque fois différentes par l'étendue du corpus, certains textes apparaissant ou disparaissant au gré de l'éditeur, et n'offrant souvent pas le même déchiffrement. Ainsi, pour ne prendre qu'un seul exemple, dans le texte de la séance du 1er mars 1855 édité en 1968, «la lueur dans le salon ${ }^{4}$ » relevée par Victor Hugo à la suite d'une observation de ses fils devient, dans l'édition Folio appelée à faire autorité puisqu' elle est la seule établie à partir du manuscrit, «la lune dans le soleil5» (2014) - invention dont la poésie aurait certes plu à Charles Trenet, mais qui n'a aucun sens.

Chacune de ces éditions mentionne des séances «tenues chez Edmond Leguével ${ }^{6}$ », dont il existe, pour certaines, des copies à la Maison de Victor Hugo, mais aucune ne donne de précision sur ce personnage, ni sur les circonstances dans lesquelles elles se sont déroulées. Aucune n'a exploité le manuscrit où sont enregistrées ces séances auxquelles participait un groupe constitué autour d'Edmond Leguével et Xavier Durrieu, proscrits de passage à Jersey. La remarquable exposition de la bibliothèque Sainte-Geneviève consacrée en 1985 par Françoise Duplessy à «Victor Hugo et le spiritisme» avait, entre autres choses, attiré l'attention sur ce manuscrit inédit, prêté pour l'occasion par la médiathèque de la ville de Saint-Girons (Ariège). Né en 1814 dans la région, élevé dans une atmosphère cléricale, Xavier Durrieu avait fait à Paris une carrière de journaliste. Il s'était imposé dans les revues les plus prestigieuses comme spécialiste notamment des questions religieuses et philosophiques. Élu en 1848 représentant de l'Ariège à l'assemblée constituante, il avait siégé à l'extrême gauche. Après la dissolution de l'assemblée, il était retourné

\footnotetext{
1. Chez Victor Hugo, Les tables tournantes de Jersey, procès-verbaux des séances présentés et commentés par Gustave Simon, Paris, Louis Conard, 1923 [désormais Gustave Simon].

2. «Procès-verbaux des séances des tables parlantes à Jersey », texte établi par Jean et Sheila Gaudon, Euvres complètes de Victor Hugo, édition chronologique publiée sous la direction de Jean Massin [désormais CFL], t. IX, Paris, 1968, p. 1165-1490.

3. Victor Hugo, Le Livre des Tables, Les séances spirites de Jersey, éd. Patrice Boivin, Paris, Gallimard, coll. «Folio classique», 2014 [désormais Folio].

4. CFL, t. IX, p. 1458.

5. Folio, p. 524.

6. Gustave Simon, p. 61. CFL, t. IX, p. 1233 et 1238.
} 
au journalisme et avait fondé La Révolution, qui fut, avec L'Avènement du peuple (le journal de Victor Hugo et de ses fils ayant pris la suite de L'Événement), l'un des derniers organes de l'opposition à survivre aux amendes et aux peines de prison infligées par le pouvoir du prince président. Contrairement à L'Avènement du peuple, La Révolution était très antiparlementaire et ne ménageait pas ses critiques à l'égard des députés de la Montagne, Victor Hugo compris. Cité à comparaître le 1er décembre 1851, Xavier Durrieu avait échappé aux Assises grâce au coup d'État. Arrêté, déporté, incarcéré puis finalement exilé, il s'était réfugié à Londres d'où il avait publié en connaissance de cause son Histoire de la persécution de décembre ${ }^{7}$, qui lui avait valu une grande popularité dans les milieux de la proscription. Du 2 septembre au 15 décembre 1853, il avait fait étape à Jersey avant de rejoindre l'Espagne, sa destination finale. Il était accompagné d'Edmond Leguével, avocat né en 1823 dans le Morbihan d'une famille aisée, un des rédacteurs de La Révolution, franc-maçon actif et militant. Condamné le 30 octobre 1851 à 3000 francs d'amende et dix-huit mois de prison pour diffamation à la suite d'un article sur les malversations que l'on attribuait au préfet de police Carlier, il avait semble-t-il pris la fuite à Londres pour échapper à sa peine. Xavier Durrieu l'avait ensuite rejoint. Charles Hugo était à trois ans près le contemporain d'Edmond Leguével, qu'il avait fréquenté en France lorsqu'ils étaient tous deux journalistes, ainsi que son frère François-Victor et Auguste Vacquerie; l'exil commun à Jersey renforçait leurs liens.

Il y a au moins trois raisons de s'intéresser au manuscrit Durrieu des séances de tables tenues chez Leguével: outre les informations précieuses qu'il apporte sur le climat idéologique qui régnait alors dans les milieux de l'exil, il fournit la preuve incontestable que des séances consignées sur des copies non référencées conservées à la Maison de Victor Hugo, dont la valeur, voire l'authenticité ont été parfois mises en doute 8 , ont effectivement eu lieu; il permet d'améliorer l'établissement du texte en révélant l'existence de variantes inconnues jusqu'ici par rapport au texte publié; il éclaire les conditions matérielles dans lesquelles était élaboré le compte rendu des séances, dont Victor Hugo avait prévu la publication posthume.

Ce manuscrit se trouve à la bibliothèque municipale de Saint-Girons; il appartient au fonds Duclos, du nom de l'abbé qui, chargé de gérer les papiers laissés par Xavier
Durrieu après sa mort à Barcelone en 1868, les lui a confiés. La cote $\mathrm{C}$ 174-12 rassemble des documents ainsi désignés, inexactement, par l'abbé Duclos : «spiritisme, Jersey, 1853-54 : notes prises par Xavier Durrieu au cours de séances de spiritisme chez Victor Hugo. 1 liasse, 60 pages ».

Sur ces soixante folios, une quinzaine seulement concerne des séances de tables parlantes tenues à Jersey; les autres se rapportent à celles qui ont eu lieu en Espagne, au moins jusqu'en octobre 1854. La chemise qui regroupe l'ensemble porte pour titre : «Mystères des tables ». En dessous, Xavier Durrieu a ajouté cet exergue : «Les exilés, les malheureux ont besoin de causer avec les esprits transmondains». Une autre main a ajouté en dessous, sous un trait : «de Jersey / Chez Victor Hugo ». Il s'agit de feuilles d'un papier de qualité médiocre pliées en deux pour composer une sorte de cahier sans reliure de $12 \mathrm{~cm}$ sur 19 environ, à quoi s'ajoutent deux feuillets libres. Toutes les pages sont utilisées recto et verso. Les conversations sont inscrites en lignes serrées, sans marges, chacune étant séparée de la suivante par deux petits traits seulement (équivalant au signe $«=»$ ). Pour les séances remarquables où interviennent des personnalités considérables qui ne déclinent pas leur identité, la mention «Robespierre » ou «Jésus », par exemple, a été portée après coup en haut à gauche. Un trait surtout est à signaler, car il a servi de motif ou de prétexte à ne pas exploiter ce manuscrit : son caractère lacunaire. Dans les propos des intervenants «transmondains», les mots sont pour la plupart tronqués, amputés des dernières lettres, notamment des morphèmes grammaticaux, ou réduits à quelques lettres initiales, voire à l'initiale seule. Des espaces blancs sont réservés pour compléter après coup les mots abrégés ou restaurer ceux qui ont été omis, ce qui n'a pas été fait. Les questions posées par les participants sont traitées encore plus cavalièrement, réduites le plus souvent à un mot caractéristique censé en indiquer la teneur générale, et quelquefois à rien : dans ce cas, l'espace laissé en blanc pour en rétablir le texte après coup donne seul idée de leur longueur. La restitution du texte intégral est donc difficile, souvent conjecturale, voire impossible. Le contexte permet de «traduire» les mots «la c dep

7. Le Coup d'État de Louis Bonaparte. Histoire de la persécution de décembre. Événements, prisons, casemates et pontons, Bruxelles, impr. de Briard, 1852.

8. Voir Folio, p. 46. 
de J est p et pard » en «la couronne d'épines de Jésus est pitié et pardon»; en revanche l'énoncé noté «Dieu est la $\mathrm{p}$ de la $\mathrm{n} d u \mathrm{t}$ » serait impossible à reconstituer en «Dieu est la planète de la nuit du tombeau» sans l'existence d'une copie conservée à la Maison de Victor Hugo, sur laquelle a été établi le texte du compte rendu publié en $1968^{9}$.

Le nombre de séances datées est de douze, mais certaines, en particulier les premières, ne sont pas datées. $\mathrm{Au}$ total on peut en identifier quinze ou seize. Les dates inscrites vont du 1er octobre au 11 décembre (1853), avec une interruption complète pendant le mois de novembre, observable aussi dans les comptes rendus du groupe Hugo. Lors de ces séances ont eu lieu quatre-vingt-une conversations, dont dix-huit sont communes au manuscrit Durrieu et au corpus de l'édition de 1968. Les intervenants sont très divers, allant d'un Huron des grands lacs à Satan et Jésus-Christ. Quelques-uns apparaissent plus d'une fois : Jean-Jacques Rousseau (quatre fois, dont deux conversations communes aux deux séries de comptes rendus), la mère de Xavier Durrieu (cinq fois, dont une commune), Marat (trois fois, dont deux communes), Socrate (deux fois, communes), André Chénier (deux fois, dont une session, commune, chez Victor Hugo : c'est l'unique compte rendu du manuscrit Durrieu qui concerne une séance tenue chez Victor Hugo).

Le manuscrit offre deux listes des participants. La première apparait le 1er décembre, à la fin d'une conversation avec Jésus. Il s'agit du jeu des portraits, auquel on se livrait quelquefois aussi chez les Hugo :

$\begin{array}{ll}\text { Ch[arles] Hugo : } & \text { - rêverie } \\ \text { F[rançois] V[ictor] h[ugo] } & - \text { peur de lui-même } \\ \text { Mme L[eguével] } & - \text { joie d'enfant } \\ \text { Ed[mond] L[eguével] } & - \text { vision } \\ \text { Thé[ophile] G[uérin] } & - \text { modestie de cœur } \\ \text { X[avier] D[urrieu] } & - \text { indécision }\end{array}$

La seconde liste figure sur le dernier folio du manuscrit, à la fin de la dernière session, celle du 11 décembre : ce sont les signatures autographes de tous ceux qui s'engagent à garder le silence sur les mystères dont ils ont eu communication. Comme il s'agit d'une sorte d'engagement contractuel, les noms sont écrits en entier :

Quennec10 Charles Hugo

Xavier Durrieu

\author{
Ed [trois points] Le Guevel11 \\ Victor Hugo fils \\ Théophile Guérin 12 \\ Rose Leguevel \\ née de Sernetz ${ }^{13}$ \\ Adèle Victor Hugo \\ Auguste Vacquerie Victor Hugo \\ A. Victor Hugo
}

L'adjonction de quatre membres du groupe Hugo (Victor Hugo, sa femme, sa fille et Auguste Vacquerie) à la première liste ne signifie pas qu'ils ont tous participé régulièrement aux séances «chez Leguével » : la seule où leur présence est attestée est celle du 10 décembre, consacrée à Socrate et André Chénier, qui se tient chez Victor Hugo ; mais Xavier Durrieu seul y représente le groupe formé autour de lui et il ne prend pas de notes ${ }^{14}$. Jamais Leguével ni Rose («Mme Leguével») ne mettront les pieds chez les Hugo.

La comparaison entre la liste du 1 er et celle du 11 décembre éclaire toutefois le fonctionnement d'un groupe dont la raison d'être est l'évocation des messagers «transmondains »: elle retrace l'évolution au terme de laquelle Edmond Leguével, à qui est associée la «vision» dans la première liste, est déchu de cette fonction au profit de Charles, porte-parole de son interlocuteur privilégié, l'Ombre du sépulcre, qui le sacre officiellement comme l'unique «voyant». C'est ainsi que le médium du groupe Durrieu s'efface derrière celui du groupe Hugo après

\footnotetext{
9. CFL, t. IX, p. 1239.

10. Quennec faisait partie de l'entourage breton d'Edmond Leguével. On ne sait pas grand-chose sur lui, et on perd sa trace après le 11 décembre 1853 .

11. Ces trois points ne sont pas une abréviation comme dans la liste précédente mais bien un rappel de son appartenance militante à la francmaçonnerie. Il faisait tant de prosélytisme que, dans le jeu des portraits, l'indécision attribuée à Xavier Durrieu fait allusion aux refus polis opposés à ses démarches insistantes pour le convertir.

12. Journaliste socialiste proche du groupe Durrieu, Théophile Guérin s'agrégera au groupe Hugo après le départ de Xavier Durrieu pour l'Espagne; il sera alors assidu aux séances de tables.

13. Née en Lorraine en 1828, Rose de Sernetz n'était pas encore à proprement parler Mme Leguével. Mère d'une petite fille, elle avait suivi Edmond en Angleterre, mais ne l'épousera dans une mairie parisienne qu'à son retour d'exil, le 9 juin 1863 .

14. CFL, t. IX, p. $1251 s q$.
} 
avoir été probablement formé par lui, qui avait été initié par Mme de Girardin à Jersey.

Sans les copies conservées à la Maison de Victor Hugo sur lesquelles le texte des éditions a été établi, certains comptes rendus du manuscrit Durrieu ne seraient pas déchiffrables. Inversement, ce dernier apporte des informations précieuses sur l'élaboration des comptes rendus publiés. En effet, le manuscrit Durrieu est un document brut qui n'a subi aucun traitement et qui n'a été conservé, sans intention de publication, que pour sa fonction mémorielle et testimoniale, signifiant doublement «J'y étais » et «C'est vraiment arrivé». La seconde de ces fonctions est aussi celle des textes que réunissait le Livre des tables, en particulier le manuscrit conservé à la Bibliothèque nationale de France sous la cote Naf 16434, auquel sont parfois annexées, collées, des «pièces justificatives» venant à l'appui de longs commentaires, par exemple, le 8 mars 1855, un article découpé dans The Illustrated London News $\left(\mathrm{f}^{\circ} 8 \mathrm{v}^{\circ}\right)$, que ne mentionne aucune édition.

Le premier constat est que les deux séries de comptes rendus opèrent chacune une sélection différente. Ainsi, pour la séance du 1er décembre, le manuscrit Durrieu enregistre les conversations, absentes du compte rendu publié, avec «une personne qui se dit Napoléon III endormi», puis une autre avec « une personne qui se dit Nicolas [Irr] endormi » - ne faisant donc pas mystère de son scepticisme - et refuse carrément de noter les propos d' «une autre personne qui se dit habitant de Jupiter» en indiquant : «résultat insignifiant». Le compte rendu du groupe Hugo enregistre de son côté pieusement les paroles mémorables de «Tyatafia» (l'habitant de Jupiter), mais ignore les deux autres communications, ne gardant que celles de Marat et de feu Mme Durrieu ${ }^{15}$. Le 2 décembre, entre les propos de Charlotte Corday et ceux de Marat ${ }^{16}$, le document du groupe Hugo omet six conversations : «Mater Durrieu, Robespierre, Jésus, Hudson Love, Judas, mère». Les 3 et 5 décembre, il néglige encore quatre intervenants, dont les conversations sont assez brèves et parfois très provocantes : «Lesurques, Lacenaire, La mort, Odry», ne gardant que JeanJacques Rousseau ${ }^{17}$. Les comptes rendus publiés résultent donc d'une sélection, opérée dans une matière beaucoup plus foisonnante encore que ce qui nous est donné à voir, selon des critères qui ne nous apparaissent pas clairement, mais qui laissent percevoir la volonté de confectionner un manuscrit présentable à un imprimeur.

\section{Marat}

Un cas particulier se présente avec l'entretien entre Victor Hugo et Marat : ignoré de Gustave Simon, il est publié par Henri Guillemin dès $1952^{18}$, oublié dans un premier temps puis enfin rattrapé au vol et donné en «appendice» de l'édition du Club français du livre, avec un texte de présentation très laconique de Jean Gaudon qui le dit «difficile à dater» et ne précise pas s'il s'agit d'un original ou d'une copie, ni de quelle main est le document ${ }^{19}$. Le manuscrit Durrieu comporte un compte rendu fragmentaire de cette conversation (que Jean Gaudon appelle «séance») qui est précieux pour diverses raisons. La date, non inscrite, mais déterminable par la place dans le manuscrit, entre une séance du 3 décembre et une autre du 7, est désormais connue grâce au Journal d'Adèle Hugo : la fille de Victor Hugo y résume, à la date du 6 décembre 1853, le récit et les commentaires de son père au sujet de sa rencontre la veille, avec Marat, «chez Leguével 20 ».

Le manuscrit Durrieu apporte deux certitudes incontestables : 1) le document publié par Henri Guillemin est authentique, en ce sens qu'un dialogue a bien eu lieu entre un personnage nommé Marat et Victor Hugo interprétant son propre personnage ${ }^{21}$ devant un public qui lui était a priori plutôt hostile; 2) l'extrait du procès-verbal conservé par Hugo est le résultat d'un processus assez complexe, que l'on peut partiellement reconstituer. Si défectueux qu'il soit, le verbatim de Durrieu a en effet le mérite incontestable d'être fidèle: les divergences que l'on constate entre son texte et celui que Victor Hugo avait conservé prouvent que des corrections ont été apportées après coup.

15. CFL, t. IX, p. 1234

16. Ibid., p. 1235-1236.

17. Ibid., p. 1238.

18. «Victor Hugo interroge les esprits »; Médecine de France, $\mathrm{n}^{\circ} 37$, novembre 1952 , p. 33-40.

19. CFL, t. IX, p. 1484 .

20. Le Journal d'Adèle Hugo, éd. Frances Vernor Guille, Paris, Lettres modernes-Minard, coll. «Bibliothèque introuvable» [désormais Le Journal d'Adèle Hugo], t. II, 1971, p. 407.

21. En insistant sur le «caractère foncièrement spectaculaire de la parole que les tables profèrent», Daniel Sangsue ajoute : «Le protocole d'enregistrement apparente les procès-verbaux à des textes dramaturgiques [...]» («Scènes de tables : Hugo et les tables parlantes», Spectacles de la parole, dir. Hélène Millot et Corinne Saminadayar-Perrin, Saint-Étienne, Éditions des cahiers intempestifs, coll. «Lieux littéraires», 2003, p. 225-242). 
On trouvera ci-après (p. 104-105) une reproduction en vis-à-vis des deux versions, qui fait apparaître les différences. Dans la version Durrieu, on a mis en caractères gras et on a souligné les passages qui sont absents de la version Hugo (Club français du livre, 1968), pourtant beaucoup plus développée. Dans cette version Hugo, on les a ajoutés aussi en caractères gras et soulignés, et on a mis en caractères gras non soulignés les passages absents chez Durrieu.

À la quatrième ligne de la version Durrieu, la didascalie «(après [un silence])», suivie d'aucun espace libre, signale un intervalle de temps entre la question («aurais-tu dem[andé] ma t[ête]?») et la réponse. La version Hugo intègre à cette place un ensemble complexe de questions et de réponses (ou de refus de répondre) qui occupe une douzaine de lignes. Si Durrieu avait omis cet échange animé où Marat, d'abord sur la réserve, finit par céder et répondre à un Hugo de plus en plus pressant, on peut supposer qu'il aurait laissé, selon son usage courant, un espace suffisant pour le restituer après coup.

À la sixième ligne, après les mots «la t[ête] qui la niait», la version Durrieu ajoute la phrase «L'int[ention] const[itue la] diff[érence]», suivie d'un blanc correspondant à une dizaine de lettres. La version Hugo omet cette phrase.

À la huitième ligne, dans la réplique de Marat qui commence par les mots «Cert[ains] hist[oriens] se mett[ent] à l'hist[oire]», la version Durrieu porte «com[me] les vers aux gr[ands] cad[avres]»; dans la version Hugo, l'adjectif «grands » a disparu.

Aux onzième et douzième lignes, après les mots «lum [ière] sur les tiennes », la version Durrieu continue par une phrase omise dans la version Hugo : «Je me cache dans le tomb[eau] du pas[sé] : tu te lèv[es] dans le berceau de l'avenir.»

À la treizième ligne, après l'exclamation « oui, le monde lib[re] ! », la version Durrieu continue avec l'injonction «Montez au cr[éneau]» que la version Hugo supprime, ne conservant que : «Peuples ressuscités, votre calvaire finit, votre évangile commence!»

Enfin, entre cette prophétie enflammée et la réplique finale de Marat, dont le texte est identique dans les deux documents, Durrieu a réservé un espace pour une réplique de Hugo qu'il n'enregistre ni ne résume. Dans la version Hugo, un texte de neuf lignes prend sa place.

Ces variantes prouvent que le manuscrit conservé dans les archives de Victor Hugo, édité par Henri Guillemin en
1952, n'est pas un verbatim des échanges, mais un texte révisé et mis au point. Elles tendent en partie à accentuer la tension dramatique des conversations. Elles attribuent à Victor Hugo des répliques très élaborées, dont on peut parfois penser qu'un texte plus court a pu être lu ou prononcé par l'interlocuteur, puis développé par écrit et inséré par la suite dans le compte rendu. Les didascalies contribuent singulièrement à cet effet, d'une façon très comparable à celles qui figurent dans les comptes rendus de séances parlementaires, sous des formes parfois très différentes suivant que l'on consulte la relation officielle du Moniteur, les versions publiées par L'Événement ou les éditions en fascicules des discours de Victor Hugo ${ }^{22}$. Elles ont aussi un autre rôle, qui est propre aux séances de la table : c'est d'attester la réalité du phénomène magnétique. À cet égard la table est traitée dans le Livre des tables exactement comme un acteur à part entière : les didascalies indiquent qu'elle a une élocution variable, donc expressive (trait ici limité à un long silence), et dans certains cas même une gestuelle. Le manuscrit Durrieu montre qu'il faut considérer sérieusement l'hypothèse que certaines de ces indications aient pu être ajoutées ou développées après coup lors de la mise au point du compte rendu.

Les variantes les plus fréquentes sont toutefois les corrections stylistiques. Elles sont relativement nombreuses dans ce texte exceptionnel, car il est l'exemple d'un exercice de haute virtuosité qui consiste à produire un texte «à la manière » de Marat, mais portant de fortes marques du «style Victor Hugo ». Les parentés stylistiques entre les deux orateurs qui dialoguent grâce à la table permettent d'atténuer leurs oppositions idéologiques. Ce qui a été éliminé par le compte rendu définitif, ce sont les surcharges, comme telle volonté d'explicitation («l'intention fait la différence »), mais surtout ces antithèses métaphoriques systématiquement associées à Hugo : la phrase «Je me cache dans le tombeau du passé, tu te lèves dans le berceau de l'avenir » faisait à ce titre l'effet d'une parodie presque grotesque. Refusée aussi la tentative, plus subtile, d'accoler

22. Pour un exemple ponctuel de ces différences, voir notamment JeanMarc Hovasse, «Déconstruire pour instruire? Quelques remarques sur une page manuscrite d'un discours de Victor Hugo à 1'Assemblée », Genesis, $\mathrm{n}^{\circ} 39$, «Avant-dire, Genèse écrite des genres oraux », dir. Gilles Philippe, 2014, p. 165-168. 


\section{[5 décembre]}

champ de $b \quad$ pas de notre siècle.

région sereine, toi au-delà de la vie, moi au-dessus

Dans la première révol aurais-tu dem ma $\mathrm{t}$ ?

- (après [un silence]) - En 93, tu t'appelais Danton; je te tutoyais, nous étions la tête de

la rév .je nai j dem la $\mathrm{t}$ qui porte l'idée, j'ai

dem lat qui la niait. - L'int const diff

- - Cert hist se mett à l'hist com les

vers aux gr cad ; ils y s la pourr quiv jug la mort.

je lais mes os à la verm $\quad$ : je do $\mathrm{m}$ â à la vér. Oui, tu as rais, tu cont

mon œuv. le s que j'ai aux

mains se f lum sur les tiennes. je me cache dans

le tomb du pas : tu te lèv dans le berceau de l'avenir.

oui, l'Eur libre! oui, le monde lib.. ! Montez au cr peup

ressus, votre calv finit; votre év commence!

- oui les dictat sont les restes

des ch bris parles rév et qui s'attach à leurs mains

la révol fut n'aura pas bes de dictature; elle sera tell

forte qu'elle pourra ê lib : elle dira à la lib : Viens et les

$\mathrm{p}$ sagen dev les deux archan réc

et deb à la porte du ciel!

La mort.

- Duperie républicaine

- je suis la mort; je tue même l'espérance

Manuscrit Durrieu, folio $11 \mathrm{r}^{\circ}$ 
champ de bataille. C'est le pas de notre siècle. Au siècle prochain la guerre aura disparu, comme l'échafaud. En attendant et dès à présent - pour bien préciser et pour marquer la différence qu'il y a entre les hommes qui remplissent ta fonction et les hommes qui remplissent la mienne - je vais t'adresser une question en deux parties. Nous causons tous les deux dans la région sereine, toi au-delà de la vie, moi au-dessus. La question que je vais te faire n'a donc rien d'amer dans mon cœur et ne doit pas te troubler. Je t'en ai dit le but. En voici la première partie : si j'avais vécu dans la première révolution, aurais-tu demandé ma tête? Veux-tu répondre?

- Non.

- La deuxième question t'aidera à répondre à la première. Tu feras une réponse collective; j'aurais mieux aimé une réponse successive. Mais soit! Voici la deuxième partie : si tu vivais dans la révolution future, demanderais-tu ma mort? - Non.

- Tu réponds que tu ne demanderais pas ma mort dans la révolution future. Donc tu peux répondre si tu l'aurais, oui ou non, demandée dans la première?

Silence de la table.

- Est-ce que tu refuses de répondre à cette question?

- Non.

- Eh bien, dans la révolution passée, aurais-tu demandé ma tête? Veux-tu répondre?

- Oui.

- Parle.

- En 93 tu t'appelais Danton; je te tutoyais; nous étions la tête de la Révolution. Je n'ai jamais demandé la tête qui portait l'idée; j'ai demandé la tête qui la niait.

- Tu sais que, dans ma pensée, l'histoire est entièrement à refaire. Elle est remplie de 93 faits par les rois et glorifiés par les historiens courtisans. Un seul 93 a été fait par le peuple; il a été l'objet d'un immense et unique anathème. Je suis de ceux qui, en confrontant tous les crimes du passé avec la grande expiation dont vous avez été les ministres, absolvent et, je le répète, glorifient l'expiation. C'est en donnant la mort que vous avez donné la vie. Nous, nous avons autre chose à faire. Vous avez donné la vie à la France. Nous, vos fils, nous devons donner notre âme au continent. Cette âme, c'est la République. Donc un lien profond unit nos deux ouvres, et vous devez nous comprendre, vous qui êtes morts pour la vôtre. Il ne peut y avoir entre nous aucune haine. Vous avez commencé, nous terminons. Seulement, à mesure que l'œuvre avance, le jour se fait, et ce que vous avez ébauché dans la nuit, nous le complétons dans la lumière. Voilà tout le secret de la douceur que les faux révolutionnaires du passé nous reprochent à nous, les véritables révolutionnaires de l'avenir. Veux-tu parler à ce sujet?

- Oui.

- Parle.

- Certains historiens se mettent à l'histoire comme les vers aux grands cadavres; ils sont la pourriture qui veut juger la mort. Je laisse mes os à la vermine; je donne mon âme à la vérité. Oui, tu as raison, tu continues mon œuvre; le sang que j'ai aux mains se fait lumière sur les tiennes. Je me cache dans le tombeau du passé, tu te lèves dans le berceau de l'avenir. Oui, l'Europe libre! Oui, le monde libre! Montez au créneau Peuples ressuscités, votre calvaire finit, votre évangile commence! - Tu viens de prononcer deux fois ce mot : Libre, et, à ce sujet, comme il est impossible que vous, qui êtes dans la lumière de la mort, vous ne voyiez pas toute la clarté de l'avenir, je vais te faire une question sur un point qui me tourmente. La patrie est sainte, mais la liberté est plus sainte encore. C'est ce qui fait que Spartacus est plus grand que Léonidas. Eh bien, dans la Révolution future, dans la révolution d'Europe, je m'habitue à cette pensée que la France doit se fondre dans l'unité des peuples, que l'idée de patrie doit s'affaiblir en se généralisant. Mais j'entends dire autour de moi que, dans le rude passage de l'état présent à l'état futur, la liberté aussi devra peut-être être voilée. C'est là ce qui me préoccupe. Je ne voudrais pas, pour la liberté, même une éclipse d'une heure. Rien ne doit s'interposer entre cet astre et l'homme, rien, pas même une révolution. Je te demande donc : aurons-nous la révolution d'Europe sans dictature et par la liberté? La liberté sera-t-elle le but? Sera-t-elle le moyen?

- Oui, les dictatures sont les restes des chaînes brisées par les révolutions et qui s'attachent à leurs mains. La révolution future n'aura pas besoin de dictature : elle sera tellement forte qu'elle pourra être libre. Elle dira à la liberté : Viens ! et les peuples s'agenouilleront devant les deux archanges réconciliés et debout à la porte du ciel. 
dans la même phrase des marques caractéristiques des deux auteurs, comme l'injonction «Montez au créneau», qui paraphrase le «Montez sur la tour, sentinelles!» du poème «Stella» (Châtiments, VI, 15), et ces «peuples ressuscités » qui font allusion à la plus célèbre harangue de Marat, celle prononcée le 1er juillet 1793 à l'Hôtel de Ville («Levez-vous donc, peuple souverain »). Tous ces biblismes tendent en tout cas à rapprocher la rhétorique de Marat, qui avait donné comme titre à son journal L'Ami $d u$ peuple, de celle du poète qui avait adopté pour devise du sien «tendre et profond amour du peuple». Dans ce contexte où l'enjeu était de faire coexister, malgré leurs différends, les proscrits socialistes et les démocrates sociaux, les problèmes de stylistique revêtaient immédiatement une dimension idéologique.

\section{Autres variantes}

Autre exemple de corrections stylistiques tirées d'autres séances, dans le discours de Rousseau enregistré le 3 décembre, les mots «Jour et nuit sont synonymes 23 » se trouvent dans le manuscrit Durrieu sous la forme «Jour et ciel sont synonymes». Rien de plus fréquent chez Hugo que ces jeux sur l'antithèse du jour et de la nuit, où les deux termes échangent certaines de leurs valeurs, contestant les équivalences banales présentées dans la version Durrieu (ciel et Dieu, puis nuit et terre, après jour et ciel). Mais dans la version Hugo cette marque de fabrique de la poésie hugolienne est travestie en un oxymore brutal et absurde. Il ne s'agit pas d'idéologie, mais de l'invention d'une poétique «surnaturaliste» qui s'épanouira plus tard dans les productions de la table. On voit qu'elle n'est pas due à un délire inspiré, mais à un calcul délibéré.

D'autres variantes s'expliquent par le souci, de la part des rédacteurs du Livre des tables, de présenter un texte idéologiquement correct. Telle suppression semble bien avoir pour but d'atténuer le spiritualisme exacerbé professé par l'âme de Rousseau : en parlant des «soleils de l'intelligence 24 », Rousseau ajoutait dans la version Durrieu «ils ne brillent que dans la mort». Cette précision trop explicite est supprimée; elle se dissimulait déjà sous cette métaphore présente dans les deux versions, dont la parfaite obscurité oraculaire produit de surcroît encore un bel effet de style surnaturaliste :
«Dieu est la planète de la nuit du tombeau 25 ». Dans le même sens, le mot «civilisateurs 26 » remplace un peu plus loin le mot «révélateurs» (version Durrieu), qui portait trop la marque de la religiosité chère aux socialistes saint-simoniens, et les «calvaires » deviennent «infranchissables 27 » dans l'absolu, tandis qu'ils l'étaient plus précisément «aux vivants » dans la version Durrieu.

Il faut enfin évoquer le cas des vers dictés par André Chénier, pour compléter ses idylles et épigrammes fragmentaires. Le manuscrit Durrieu fait apparaître que la version publiée par le Club français du livre est une mise au net, mais la transcription plus que lacunaire de Durrieu, apparemment peu intéressé par les amours de Néère et de Chromis, donne aussi quelques exemples de vers refaits et effacés du compte rendu. Ainsi y trouve-t-on cette ébauche où l'alternance des rimes est respectée, à défaut du rythme de l'alexandrin («Néère veut mourir, Chromis infidèle/Va demander à d'autres femmes qu'elle»), tout l'inverse de la version refaite : «Néère veut mourir. Les sylphes dans les bois/ Font silence : l'écho veut entendre sa voix 28 ». On relève enfin, et surtout, pour les séances des 10 et 11 décembre, ces deux indications précieuses : «corrections dans cette pièce [...] charge $\mathrm{Ch}$ Hugo »; «aussi corrections dans cette pièce $([\ldots]$ chez $\mathrm{V}$. Hugo)». La première seule est ainsi développée, et édulcorée, dans la version Hugo : «- Qui charges-tu de continuer ce travail? - Charles Hugo ${ }^{29}$.» C'est avouer que les textes publiés ont été mis au point, à tête reposée mais toujours inspirée, dans la chambre de Charles. Ce sont les failles inévitables d'une improvisation en vers, même préparée. Dans la séance du 30 mars 1854, comme dans quelques autres, une «véritable mise en scène

23. CFL, t. IX, p. 1238. La réplique de Rousseau commence ainsi : «Jour et nuit sont synonymes. Ciel et Dieu sont le même mot. Nuit et terre sont synonymes; homme et doute sont le même mot. »

24. Ibid., p. 1239.

25. Ibid.

26. Ibid., p. 1242.

27. Ibid.

28. Ibid., p. 1254. Ces rimes masculines y suivent deux rimes masculines (fraisier/rosier), alors que la table respecte habituellement toujours la règle élémentaire d'alternance de la prosodie classique et romantique. La version Durrieu permet d'imaginer comment cette faute aurait dû être corrigée.

29. Ibid., p. 1250. 
de la rature $^{30} \gg \mathrm{y}$ remédiera plus habilement : ce jour-là, le lion d'Androclès dictera puis acceptera de corriger ses strophes en fonction des arguments avancés par Victor Hugo pour éviter des rencontres de mots malencontreuses ou des ruptures de sens 31 .

Le manuscrit Durrieu sert ainsi de document de contrôle. Il présente une version des séances à l'état brut, telles qu'elles se sont déroulées réellement, et révèle le travail de correction qui a été fait lors de la mise au net.

\section{Tachygraphie ou cryptographie?}

Cette interaction entre la table ou le médium et son public va sans doute bien au-delà de cette collaboration visant à surmonter les problèmes de versification. Elle semble intervenir très en amont, dans la constitution même du message. On ne peut manquer de s'interroger sur la nature et la signification de cette pratique qui consiste non à abréger, mais à tronquer systématiquement les mots. La première hypothèse qui vient à l'esprit est qu'il s'agit d'une sorte de tachygraphie, comme en utilisaient des journalistes dont la fonction était de rendre compte des séances de l'Assemblée. Les comptes rendus «hugoliens » des premières séances de table, moins soignés que les suivants, conservent encore des traces qui prouvent l'usage de telles abréviations dans le groupe. Ainsi, le 15 septembre 1853, on trouve «Mad. $\mathrm{H}^{32}$.» pour «Madame $\mathrm{H} u g o »$; plus difficile le lendemain, «mon temp ${ }^{33}$.» pour «mon tempérament». Un indice inverse, et peut-être encore plus sûr, atteste que le secrétaire chargé de la mise au net était préparé à compléter des mots tronqués. En effet, s’il n'était pas rompu à cette tâche de manière un peu mécanique, habitué par exemple à compléter systématiquement les fins de mot en -ris par -te, on ne comprendrait pas pourquoi on trouve au moins à deux reprises le mot «Phalariste ${ }^{34}$ », certainement pas dicté par la table, qui parlait dans les deux cas du tyran Phalaris et de son fameux taureau.

Le manuscrit Durrieu permet donc d'appréhender un peu mieux la complexité et la précision du dispositif mis en place pour produire ces textes. Comme tout message, ils ont un émetteur, immatériel, qu'il convient de laisser en dehors des investigations. C'est «un tiers», comme le dit Charles Hugo dans une de ses rares tentatives d'élucidation ${ }^{35}$. Ce message a la caractéristique d'être présenté sous une forme numérique (une suite de nombres); le «médium» est le premier chaînon de la réception; il est obligatoirement associé à un second qui convertit cette suite de nombres en littéral oralisé, traduisant une séquence de sons par une lettre qu'il dicte. S'il se borne à ce rôle, le résultat est un ruban de lettres sans accents, sans apostrophes, sans majuscules, sans ponctuation, et surtout sans signes de démarcation de mot, un peu comme se présente un manuscrit antique. Beaucoup de chercheurs l'ont remarqué, en frappant intégralement à la suite la totalité des lettres constituant le message, il aurait été rigoureusement impossible d'écouler dans le temps donné un trafic aussi volumineux que celui des grandes sessions ${ }^{36}$. En fait la fonction du second est plus importante et plus délicate : elle consiste aussi à segmenter la chaîne des lettres en fonction des mots qu'il parvient à identifier. Ce que Charles appelait «deviner 37 ».

La troncation dans le manuscrit Durrieu peut donc s'expliquer si cette opération avait lieu avant même que la dictée du mot soit terminée : une fois «deviné» le mot qui est en cours de frappe, l'émetteur (théoriquement omniscient) en est informé, et cesse ses frappements dès que le message est désambiguïsé, pour passer immédiatement au mot suivant. C'est cette procédure qui dans une séance du groupe Leguével crée un incident lors de l'apparition de Saint-Arnaud, maréchal de Napoléon III : à partir de «nomdeteste», le «second» a pratiqué une coupure de mot inadéquate en croyant deviner «nom de Teste» (Jean-Baptiste, le pair de France corrompu mort en 1852),

30. Daniel Bilous «Écrits sur tables, Les au-delà intertextuels de l'exil», Idéologies hugoliennes, éd. Anne-Marie Amiot, Nice, Éditions Serre, 1985, p. 99.

31. Voir CFL, t. IX, p. 1347.

32. Ibid., p. 1214

33. Ibid., p. 1218.

34. Ibid., p. 1261 (et 1459).

35. Voir Le Journal d'Adèle Hugo, éd. citée, t. II, p. 278.

36. Le dernier en date, et le plus attentif aux détails techniques, est Naoki Inagaki, peut-être parce qu'il est familier d'une culture moins effarouchée par les fantômes que la nôtre depuis les Lumières : «Victor Hugo, auteur possible des textes des "Tables parlantes" : comparaison entre leurs Procès-verbaux et les "notes" prises par Hugo », Comment naît une œuvre littéraire? Brouillons, contextes culturels, évolutions thématiques, dir. Kazuyoshi Yoshikawa et Noriko Taguchi, Paris, Champion, 2011, p. 99-113.

37. Voir Le Journal d'Adèle Hugo, éd. citée, t. II, p. 278. 
alors qu'il fallait comprendre «nom détesté », qui annonçait Saint-Arnaud. Le manuscrit Durrieu semble donc donner raison à Victor Hugo lorsqu'il tente de montrer à son fils que le médium, quand la table commence à frapper, sait ce qui va suivre : «Tu te mettras à la table, et avant qu' elle ne parle tu te pencheras à mon oreille et tu me diras le mot qu'elle va dire ${ }^{38}$. $\gg$ Charles se garde bien de répondre à cette suggestion... car c'est au récepteur de «deviner». Quant à Durrieu, on peut supposer qu'il se borne à noter les lettres effectivement frappées, laissant au lecteur de son manuscrit le soin de combler les vides (si jamais lecteur il devait y avoir autre que lui-même), ce qui ne lui pose à lui-même aucun problème : il s'agit alors d'une sorte de cryptographie.

Il fallait en tout cas une grande proximité entre le médium et son second, qui devait avoir une bonne connaissance du champ lexical où s'inscrivait le message, pour deviner promptement ce qui allait suivre : de là sans doute les fréquents changements que notent les procès-verbaux. De là aussi l'importance des anaphores dans ces textes, puisqu'un mot apparu une fois pouvait faire retour à plusieurs reprises à l'aide de sa seule initiale : quand la guillotine (cent vingt-quatre frappements) revient, elle s'annonce comme «la g» (sept frappements). Même chose pour les antithèses : «berceau » rend par exemple prévisible «tombeau», et vice-versa.

\section{Le folio $25 \mathrm{r}^{\circ}$ du manuscrit Naf 16434}

Ces hypothèses sont confirmées par quelques séances de Marine Terrace où sont rendus manifestes, et même ostensibles, les processus décrits ci-dessus. L'exemple le plus caractéristique est celui de la séance tenue le 4 juin 1855 (manuscrit Naf 16434, f $23 \mathrm{r}^{\circ}-26 \mathrm{r}^{\circ}$ ). Ce soir-là, l'émetteur a exigé que le secrétaire, qui était Victor Hugo, enregistre la dictée dans l'obscurité complète. On peut observer le résultat sur la reproduction de la page 25 (voir fig. 1). Avant de commenter les singularités de la graphie, quelques remarques sur la production du texte lui-même, qui présente les mêmes caractéristiques que la conversation avec Marat du 5 décembre 1853. D'abord le travail de l'intertextualité39 : il s'agissait en 1853 de la confrontation entre deux mixtes textuels hautement improbables, du Marat «hugolisé »

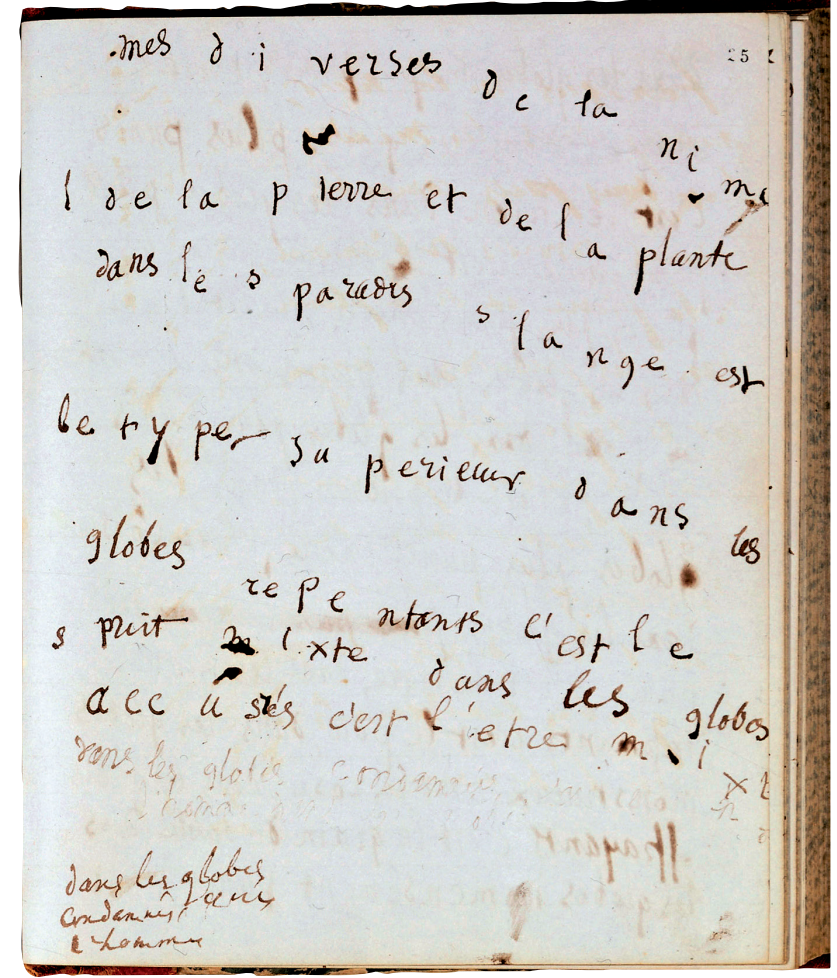

Fig. 1 : Folio 25 r $^{\circ}$, Naf 16434 (BnF, département des Manuscrits)

contre du Hugo «maratisé». L'émetteur du 4 juin se souvient quant à lui d'un poème qui traînait depuis avril 1854 dans la chambre de Victor Hugo, «À la fenêtre pendant la nuit», qui entrera dans Les Contemplations (VI, 9). Il tire de l'alliance de ces deux substantifs («Fenêtres de la nuit, ouvrez-vous...») le début de ce qu'il faut bien appeler un

\section{Ibid., p. 279.}

39. Daniel Bilous, dans l'article cité ci-dessus (note 30), en empruntant ce terme aux Palimpsestes de Gérard Genette, a défini la bonne méthode pour étudier sérieusement le corpus des Tables; mais sa tentative de l'appliquer en s'appuyant sur les intuitions de Gustave Simon et de Claudius Grillet (auteur d'un Victor Hugo spirite, Lyon/Paris, Librairie Emmanuel Vitte, 1929), dont l'expertise en matière de poétique était limitée, était une gageure intenable. L'intertextualité hugolienne tient évidemment une place importante ( nous trempons tous notre plume dans votre encrier», écrivait Vacquerie à Hugo), mais elle couvre une aire beaucoup plus vaste, qui va du journal quotidien en langue anglaise à l'article de vulgarisation scientifique (voir CFL, t. IX, p. 1450 et 1457). 
poème en prose, d'une incontestable puissance poétique. Outre les mots du titre, il emprunte à son «hypotexte» le thème général : l'angoisse devant les inconnues que recèle le spectacle du ciel étoilé. À partir de là, le texte s'engendre d'une manière mécanique par une série d'anaphores menant à des correspondances d'abord banales, puis de plus en plus extravagantes et choquantes. En somme, on y retrouve l'ensemble des procédés de fabrication de ce que les critiques décideront, quelque soixante-dix ans plus tard, de nommer les «révélations de Jersey 40 ».

L'analyse de la graphie prouve que la prise de notes de Victor Hugo est identique à celle de Durrieu, dans son principe sinon dans ses résultats. Profondément désorganisée par la nécessité d'écrire dans l'obscurité, la dictée rend très évidente la coexistence de deux graphies, l'une qui correspond aux lettres frappées, dans l'attente d'un sens, l'autre aux fins de mots «devinées », et non frappées. Ainsi «-mes», en cursive avec des lettres liées est la fin de «formes»; on a ensuite «d i V» frappé et noté en lettres séparées puis «erses» non frappé ajouté ensuite. L'introduction inattendue du thème de la chaîne des êtres dans un contexte cosmique rend impossible l'identification de «animal», avec la fausse hypothèse de «la» article, écrit en cursive, les autres lettres étant jusqu'au «l» final épelées séparément; mais dès lors que le nouveau champ sémantique a été reconnu, l'initiale «p», isolée, déclenche d'une seule traite «ierre», et le «p» suivant se complète en «lante», en écriture cursive; «p a» fait un peu difficulté avant que soit devinée la suite «r $\mathrm{r}$ adis». La stabilité du champ sémantique joue donc un rôle important : «su périeurs», «re pe ntants» sont «devinés» un peu plus vite que «a cc u sés». Mais surtout les répétitions facilitent beaucoup l'anticipation du sens. Le premier «d a n s » est épelé, pour la seconde occurrence le «d» appelle automatiquement «ans les globes» prévisible à $100 \%$ après la lettre « $\mathrm{d}$ » et donc écrit immédiatement en cursive. Tels sont les bénéfices de l'anaphore : la fluidité de la communication du texte est proportionnelle à la rigidité de sa construction.

On peut mesurer le gain en rapidité réalisé grâce à la promptitude de l'identification du mot par le second et l'auditoire, en se basant non sur le nombre de lettres, mais sur l'addition de leurs valeurs. Pour «p ar a» frappé et noté, puis «dis» deviné, l'économie est de $47 \%$; pour «p ierres», de $82,8 \%$; pour «s u p érieur», de $59 \%$; pour «p lantes», de $60,8 \%$. À ce compte de longues conversations étaient possibles en relativement peu de temps. Le tandem composé par le médium et son assistant fonctionnait donc comme un unique «récepteur», qui lui-même était en interaction permanente avec l'émetteur dématérialisé, capable, quant à lui, d'entendre les propos des récepteurs et de lire leur dictée. On demandait en somme au second d'avoir lui aussi une certaine sorte de fluide, l'oreille fine, l'intuition vive et sûre.

Dans le cas présent, en outre, on a eu recours à un double enregistrement, comme chez Leguével : une didascalie de la page 24 nous apprend que pour contourner l'interdit, Théophile Guérin est allé «dans la chambre à côté», où il pouvait s'éclairer. Nous le connaissons : c'est lui dont un esprit transmondain avait loué la «modestie de cœur» le 1er décembre 1853 chez Leguével. Victor Hugo a cessé d'écrire en bas de la page 25 , ne s'étant pas aperçu que sa plume était sèche. En bas à gauche il a noté les mots qu'il avait manqués, et fermé le cahier (ou tourné la page) alors que l'encre n'était pas encore sèche, comme on peut le voir par les marques portées sur le verso de la page 24. L'ensemble, depuis le début, est reporté au verso de la page 24 ; la suite, après l'abandon de Victor Hugo, est reportée au verso de la page 25 , puis au recto de la page 26, copiée par Victor Hugo probablement à partir d'un manuscrit de Guérin.

\section{Conclusion}

Le manuscrit Durrieu est un renfort précieux pour tous les chercheurs qui, sans être «fanatiques» des fantômes de Jersey, entreprennent d'étudier méthodiquement ces textes en s'affranchissant des questions métaphysiques qui opposaient nos grands-pères en d'âpres batailles entre rationalistes et spiritualistes. Plutôt que de débattre à perte de vue pour savoir si les spectres existent ou non, on constatera

40. Le texte semble avoir impressionné : l'éditeur de Folio en a trouvé une copie faite par Mme Hugo (Folio, p. 395 sq.). On serait tenté de saisir sur cet exemple le mode de production de variantes accidentelles. Mais Folio donne pour le compte rendu du folio 25 la leçon inexplicable «allumés» (ibid., p. 546) alors que la copie publiée donne le bon texte («accusés»). La fin aussi diffère : la copie donne «les globes qui se situent dans la nuit», ce qui n'a guère de sens, alors qu'il fallait lire «dans les globes qui se tuent c'est la nuit». Bref, impossible d'accorder un quelconque crédit à l'édition Folio pour un travail sérieux. 
avec Daniel Bilous que les textes qu' on leur attribue existent, eux, incontestablement, et sont soumis aux mêmes lois que les autres ${ }^{41}$. L'étude attentive et méthodique des manuscrits peut seule nous éclairer sur le mode matériel de leur production. C'est d'ailleurs bien ainsi que l'entendait Victor Hugo, qui a légué la totalité de ses manuscrits à la Bibliothèque nationale de France, y compris «les volumes dictés à [s]on fils Charles par la table ${ }^{42}$ »; ce n'est pas de son fait qu'ils en ont été soustraits ${ }^{43}$. En permettant l'étude comparative de deux versions de plusieurs séances, le manuscrit Durrieu procure une sorte de pierre de Rosette qui rend visibles les procédures employées pour établir le document destiné à en perpétuer le souvenir. Il montre l'importance de l'intertextualité politique et idéologique qui a présidé à la genèse du premier cahier du Livre des tables; il met en évidence le fait que Victor Hugo n'est nullement le producteur des textes attribués aux spectres, mais bien leur destinataire privilégié; il révèle la manière dont son fils Charles, après s'être imposé à un groupe qui lui était politiquement hostile, a pris le pouvoir pour devenir officiellement le «voyant» du groupe Hugo. L'étude méthodique des deux cahiers retrouvés, et spécialement du dernier, celui qui est enregistré sous la cote Naf 16434, réserve sans doute bien d'autres surprises, que l'état actuel de leur publication ne permet malheureusement même pas d'entrevoir.

41. Voir Daniel Bilous, art. cité, p. 97.

42. Voir CFL, t. X, p. 459. Il s'agit d'une note inscrite dans la marge du manuscrit du poème «Au Lion d'Androclès » (La Légende des siècles, Première Série, II). L'écriture prouve qu'elle est contemporaine des textes de William Shakespeare où Hugo revient sur l'épisode de la «table parlante» (voir CFL, t. XII, p. 170).

43. Dans l'état actuel de nos connaissances, il est impossible de dire pourquoi, quand et par qui la conversation avec Marat a été détachée de l'ensemble des copies destinées au premier cahier du Livre des tables, actuellement disparu. Prévoyant une publication posthume, Hugo n'avait rien à cacher, et il n'y a pas d'exemple qu'il ait censuré des documents éclairant les cheminements, même sinueux, de sa pensée. 
JEAN-Claude FiZAine, ancien élève de l'ENS (promotion de 1956), est professeur émérite de l'université Paul-Valéry (Montpellier 3), spécialiste de l'imaginaire religieux chez les romantiques français (Balzac, Nerval, Sand). Il a édité plusieurs textes narratifs de Théophile Gautier en Pléiade et plusieurs ouvres de Victor Hugo en Bouquins : Religions et Religion, L'Âne, La Pitié suprême, Torquemada. Il a aussi établi l'édition critique d'un manuscrit relatif aux séances spirites de 1853 : Victor Hugo et les mystères de Jersey, un manuscrit inédit de Xavier Durrieu (les séances «chez Leguével»).

jcfizaine@free.fr

\section{Pour une étude génétique du Livre des tables}

Sur les quatre cahiers manuscrits où étaient enregistrés les textes issus des séances dites spirites tenues par la famille de Victor Hugo lors de son séjour à Jersey, de septembre 1853 à octobre 1855, trois ont été édités, sans résoudre toutes les énigmes que présente cet épisode de la vie du poète. Le manuscrit conservé dans les archives de Xavier Durrieu, récemment déchiffré par Jean-Claude Fizaine, apporte un éclairage neuf et décisif sur les premières séances, du 1er octobre au 11 décembre 1853, dont les originaux des procès-verbaux manuscrits n'ont toujours pas reparu. Il met fin aux doutes quelquefois émis sur l'authenticité de certaines séances et permet, en révélant l'existence de variantes, d'améliorer l'établissement du texte. Il éclaire enfin les procédures selon lesquelles était élaboré le compte rendu des séances, que Victor Hugo avait conservé en vue d'une publication posthume.

Among the four manuscript notebooks that recorded the texts produced by the Spiritist séances held by Victor Hugo's family during his stay in Jersey, from September 1853 to October 1855, three have been published but without solving all the enigmas posed by this episode of the poet's life. The manuscript, held in Xavier Durrieu's archives and recently transcribed by Jean-Claude Fizaine, casts a new and decisive light on the first séances, held from October $1^{\text {st }}$ to December $11^{\text {th }} 1853$, the original official reports of which have still not been found. However, it dispels the doubts sometimes expressed about the authenticity of some séances and, by revealing the existence of variants, allows for a better establishment of the text. Last, it shows the procedures used to formulate the record of the séances that Victor Hugo kept for a posthumous publication.

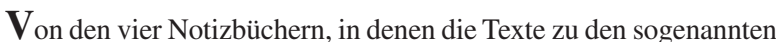
spiritistischen Sitzungen (Séancen) der Familie Victor Hugo, datierend von seinem Aufenthalt in Jersey - September 1853 bis Oktober 1855 -, wurden drei herausgegeben, ohne dass dadurch alle Rätsel gelöst worden wären, die jene Epoche des Lebens des Schriftstellers darbietet. Das Manuskript, das sich in den Archiven von Xavier Durrieu erhalten hat und das kürzlich von Jean-Claude Fizaine transkribiert wurde, birgt eine neue und entscheidende Erhellung zu den ersten Sitzungen die zwischen 1. Oktober und 11. Dezember 1853 stattfanden, und von denen die Originalprotokolle immer noch nicht aufgetaucht sind. Es setzt gewissen Zweifeln ein Ende, die manchmal bezüglich der Authenzität einiger Séancen geäußert wurden und erlaubt durch die Enthüllung der Existenz von Varianten die verbesserte Etablierung des Texts. Es erhellt schließlich die Prozeduren, gemäß denen das Protokoll der Sitzungen erstellt wurde und die Victor Hugo im Hinblick auf eine postume Publikation aufbewahrt hatte.
De los cuatro cuadernos manuscritos en los que se registraron los textos relativos a las sesiones llamadas espiritistas organizadas por la familia de Víctor Hugo durante su estadía en Jersey -entre septiembre de 1853 y octubre de $1855-$, los tres que han sido ya editados no han resuelto todos los enigmas que plantea este episodio de la vida del poeta. El manuscrito conservado en el archivo de Xavier Durrieu, recientemente descifrado por JeanClaude Fizaine, aportan un esclarecimiento nuevo y decisivo acerca de las primeras sesiones -del 1 de octubre al 11 de diciembre de 1853- cuyas actas originales manuscritas siguen desaparecidas. Este manuscrito pone fin a las dudas que han solido emitirse acerca de la autenticidad de algunas sesiones y permite, al revelar la existencia de variantes, mejorar el establecimiento del texto, esclareciendo, finalmente, los procedimientos a través de los cuales se elaboró el informe sobre las sesiones, que Víctor Hugo conservó para una publicación póstuma.

Dos quatro cadernos manuscritos em que foram registados os textos saídos das sessões espíritas realizadas pela família de Victor Hugo durante a sua estadia em Jersey, de setembro de 1853 a outubro de 1855, foram publicados três, sem resolver todos os enigmas ligados a este episódio da vida do poeta. O manuscrito preservado nos arquivos da Xavier Durrieu, recentemente decifrado por Jean-Claude Fizaine, traz uma luz nova e decisiva para as primeiras sessões, de 1 de outubro a 11 de dezembro de 1853 , de que continua a faltar o original do relato manuscrito. Coloca-se um termo a ocasionais dúvidas sobre a autenticidade de algumas sessões, enquanto a existência de variantes torna possível melhorar o texto. Finalmente, esclarece-se o processo pelo qual Victor Hugo elaborava as « actas » das sessões, tendo em vista uma sua publicação póstuma.

Tre dei quattro quaderni manoscritti sui quali erano registrati i testi delle sedute spiritiche tenute dalla famiglia di Victor Hugo durante il suo soggiorno a Jersey, dal settembre 1853 all'ottobre 1855 , sono stati pubblicati, senza risolvere tutti gli enigmi che presenta questo episodio della vita del poeta. Il manoscritto conservato negli archivi di Xavier Durrieu, decifrato di recente da Jean-Claude Fizaine, fornisce un punto di vista nuovo e decisivo sulle prime sedute, dal 1 ottobre all' 11 dicembre 1853 , i cui verbali originali non sono ancora riapparsi. Esso mette fine ai dubbi talvolta sollevati sull'autenticità di alcune sedute e permette, rivelando l'esistenza di varianti, di migliorare l'edizione critica del testo. Chiarisce, infine, le procedure di composizione del verbale delle sedute, che Victor Hugo aveva conservato in vista di una pubblicazione postuma. 